\title{
Target Tracking Based on Mean-shift and Kalman Filter
}

\author{
Songtao Jiang \\ School of Electrical Engineering, Tianjin University of Technology
}

\begin{abstract}
Keywords:target tracking; the mean deviation; Kalman filter; Kernel function histogram.
\end{abstract}
\begin{abstract}
Analysis of the scheme - shift is difficult to effectively track the main defect of gray level moving targets un-der complicated background, puts forward the combination of Mean shift and kalman filter method for target tracking. The method by using kalman filter to predict the target in the current moment of starting position, then scheme - the shift in the location of the neighborhood looking for targets within the location. At the same time, use Bhattacharyya coefficient measurement "target model" and "candidate model" similarity degree, determine whether "candidate model" is replaced by "target model", to avoid excessive update target model. On the background of object plane target image sequence test results show that this method is compared with the original scheme shift method can obviously improve the stability of target tracking.
\end{abstract}

\section{Introduction}

With visible light and infrared imaging sensor to specify the target in video image is robust and effective tracking imaging tracking system in a very challenging research field. Mean - shift algorithm is a kind of based on kernel function density increasing parameter estimation method, with its rapid matching, target deformation is not sensitive features has been widely applied in the field of target tracking. Scheme - shift, however, there are still some of the original rational is insufficient, the complicated background of gray-scale imaging moving target tracking performance also needs to be improved, such as the Mean - shift algorithm in the center of the starting place for Taylor expansion after the first two functions to approximate similarity, starting center should be as close as possible to the target location not too far apart, otherwise it will lead to trace points to stay in the local extreme of Bhattacharyya coefficient instead of the true location, where serious target with leakage, will happen even with by mistake. Moreover, target often be other objects in the scene, with low levels of target model in the moments before and after similar case still be updated, has been the target model updating problems, led to the background interference target model, target tracking performance degradation, the lack of adequate scene adaptability and robustness. According to the movement continuity, USES the kalman filter and the change of residual error to predict Mean2shift algorithm in target starting center is one of the more effective way to solve the defects of but failed to solve the problem of the target model and update the method.

This paper puts forward the combination of Mean shift and kalman filter target tracking method. The method using kalman filter to predict the target initial position, and then use scheme - shift algorithm in the location of the neighborhood looking for targets. At the same time, use Bhattacharyya coefficient measurement "target model" and "candidate model" of similar degree, and to determine whether the model of "candidate" has changed to "target model", avoid "target model" by the scene change and update.

\section{Mean shift algorithm}

\subsection{The target schema}

Target histogram model is based on the search window contains the corresponding probability density of different imaging features. Usually, far away from the center of a window pixels than closer from the center pixel belongs to target probability is smaller, and gray level probability density is a function of pixel location. Included in the search window, the first $\mu$ characteristic value of the probability density as follows: 


$$
\begin{aligned}
& \hat{p}_{u}(y)=c \sum_{i=1}^{n} k\left[\left\|\frac{y-x_{i}}{h}\right\|^{2}\right] \delta\left[b\left(x_{i}\right)-u\right] \\
& u=1, \ldots, m
\end{aligned}
$$

Type: $\mathrm{y}$ is the search window center pixel coordinates; $k\left(\|x\|^{2}\right)$ is the kernel function; $\mathrm{h}$ the bandwidth of the kernel function, the general is equal to half the width of the search Windows $\left\{x_{i}\right\} i=1, \ldots, n$ said other pixel coordinates in the search window, $\mathrm{n}$ for total pixels in the window; $b\left(x_{i}\right)$ features can be either a scalar, vector may also be a variety of features; $\mathrm{c}$ is a standardized coefficient. Function the role of $\mathrm{b}$ and the delta is whether $x_{i}$ place characteristic belongs to mu; $k\left(x_{i}\right)$ is pixel position weight distribution function, defined as follows:

$$
k(x)=\left\{\begin{array}{l}
\frac{2}{\pi}\left(1-\|x\|^{2}\right) \quad\|x\| \leq 1 \\
0 \quad \text { otherwise }
\end{array}\right.
$$

Kernel function histogram is, in fact, a kind of weighted representation of the distribution of characteristics, the weights are determined by the kernel function $\mathrm{k}(\mathrm{x})$. Mean-shift target tracking party use $\left\{\widehat{p}_{u}(y)\right\}_{u=1, \ldots, m}$ said the target model.

\subsection{Similarity measure}

For a given two images, one containing the tracking target, called "image model", another image is referred to as the "candidate images". Target tracking task is in the "candidate images" to find out the tracking target, and the accurate positioning.

Assumes that the target in the center of the "image model" of the tag is 0 , the candidate target center for $y$. By type (1) the definition of target model can determine the aim, and the histogram of the candidate target respectively $\hat{p}_{0}=\{(y)\}_{u=1, \ldots, m}$ and candidate target $\hat{p}_{u}=\left\{\hat{p}_{u}(y)\right\}_{u=1, \ldots, m}$ use Bhattacharyya coefficient as $\hat{p}_{0}$ and $\hat{p}_{u}$ similarity metric function between.

$$
\rho_{y}=\rho\left[\hat{p}_{0}, \hat{p}_{y}\right]=\sum_{u=1}^{m} \sqrt{\hat{p}_{u}(0) \hat{p}_{u}(y)}
$$

Similarity metric function $p(y)$ is actually two m unit vector $\hat{p}_{0}$ and $\hat{p}_{y}$ between the cosines value of the Angle. Obviously, when the target and the candidate targets, the greater the similarity of the Angle between the hour and $p(y)$

\subsection{The mean deviation search}

Set y0 search starting point for the current moment.py in $\hat{p}_{u}\left(y_{0}\right)$ of Bhattacharyya coefficient for the Taylor expansion to approximate and take the first two.

$$
\rho\left[\hat{p}_{0}, \hat{p}_{y}\right] \approx \frac{1}{2} \sum_{u=1}^{m} \sqrt{\hat{p}_{u}\left(y_{0}\right) \hat{p}_{u}(0)}+\frac{1}{2} \sum_{u-1}^{m} \hat{p}_{u}(y) \sqrt{\frac{\hat{p}_{0}(0)}{\hat{p}_{u}\left(y_{0}\right)}}(4)
$$

To write it on type to the following form:

$$
\rho\left[p_{0}, p_{y}\right] \approx \frac{1}{2} \sum_{u=1}^{m} \sqrt{\hat{p}_{u}\left(y_{0}\right) \hat{p}_{u}(0)}+\frac{c}{2} \sum_{i=1}^{n} w_{i} k\left[\left\|\frac{y-x_{i}}{h}\right\|^{2}\right]
$$

Among them:

$$
w_{i}=\sum_{u=1}^{m} \sqrt{\frac{\hat{p}_{u}(0)}{\hat{p}_{u}\left(y_{0}\right)}} \delta\left(b\left(x_{i}\right)-u\right)
$$


By equation (6), to make the py is the largest, to make the function $\sum_{i=1}^{n} w_{i} k\left[\left\|\frac{y-x_{i}}{h}\right\|^{2}\right]$ maximum. Through the Bhattacharyya coefficient and maximum available search window center offset for iterative formula:

$$
y_{j+1}=\frac{\sum_{i=1}^{n} x_{i} w_{i} k_{1}^{\prime}\left[\left\|\frac{y_{i-} x_{i}}{h}\right\|\right]}{\sum_{i=1}^{n} w_{i} k_{1}^{\prime}\left[\left\|\frac{y_{j}-x_{i}}{h}\right\|\right]} j=1,2, \ldots(7)
$$

By the nature of the mean deviation, the search window is always to the local extreme of Bhattacharyya coefficient (peak). When the window kernel function to peak, kernel function window will be the target of the convergence, and finish the target positioning.

\subsection{The target model updating}

Goals often be other objects in the scene, will lead to serious moments before and after the kernel function of histogram similarity is reduced, Bhattacharyya coefficient of rho $\mathrm{p}(\mathrm{y})$ decrease quickly. At this point, such as "candidate model" of the current time change for the "target model", the target model would be background interference, a model updating, the target tracking performance fell dramatically. So we must design an updated criterion function to avoid the target model.

Bhattacharyya coefficient of $\mathrm{p}(\mathrm{y})$ is to measure the "target model" and "" the candidate model similarity function, in this paper, we use it to determine whether or not to accept" candidate model "replacement for the current" target model ".p(y), the greater the "target model" and "candidate model", the more similar model of "candidate" replaceable "target model" for the current moment; On the other hand, shows that the observation model of the current time is severe interference, should not be "candidate model" to replace "target model" for the current moment. Therefore, the target model update by the following principles:

$$
\hat{p}_{0}(m+1)=\left\{\begin{array}{cc}
\hat{p}_{0}(m) & \rho(y)<\lambda \\
\hat{p}_{y}(m+1) & \rho(y) \geq \lambda
\end{array}\right.
$$

Type: $\lambda$ for threshold, $\mathrm{m}$ said discrete sampling time. Experimental results show that the gray image, lambda take 0.5 can well meet the requirements.

\section{Kalman filter prediction initial point}

Mean-shift the window shift algorithm iterative formula (7) is pie Bhattacharyya coefficient in $\hat{p}_{u}\left(y_{0}\right)$ for the Taylor expansion and take the first two approaches, Bhattacharyya coefficient (function) in the first frame of the image search starting point y0 place should have continuity, y0 should be as close as possible to the target true location not too far apart, otherwise it will lead to target tracking points stay on the local extreme of Bhattacharyya coefficient, and the local extreme value is less than the target of the Bhattacharyya coefficient, once the error accumulation to reasonably leads to target with leakage. Literature [6] to choose a moment before target tracking point search window starting point for the current moment, once the target moving too fast, the moment before target tracking point with the current moment the location there is a big distance, is likely to lead to the error.

This paper adopts kalman filter to determine the search window starting center. In consecutive frames $(\mathrm{k}-1)$, the center of the target location information formed its trajectory. The center location information as observed value of kalman filter, first the kalman filter is adopted to predict $\mathrm{k}$ time the location of the object, as the starting position of Mean2shift search window, namely the starting center, then the Mean-shift in the location of the neighborhood to find the target tracking position. After. To the target location as the observation value of the kalman filter, prediction $(\mathrm{k}+1)$ moment goal starting position. 
If working target center in the $X(m)=\left[\begin{array}{llll}x(m) & y(m) & x^{\prime}(m) & y^{\prime}(m)\end{array}\right]^{T}$, axis movement are a random disturbance of uniform motion in a straight line acceleration. The target motion state vector $\mathrm{x}$, where $\mathrm{x}(\mathrm{m}), \mathrm{y}(\mathrm{m})$ are respectively the target center in $\mathrm{x}, \mathrm{y}$ axis location component, $\mathrm{x}^{\prime}(\mathrm{m}), \mathrm{y}^{\prime}(\mathrm{m})$ is respectively in the $\mathrm{x}, \mathrm{y}$ axis. Objective observation vector $Y(m)=\left[\begin{array}{ll}x_{c}(m) & y_{c}(m)\end{array}\right]^{T}$, including XC (m), yc (m) of target center respectively in the $\mathrm{X}, \mathrm{Y}$ axis on the observed value. So, the target state equation and observation equation can be represented using the following equation:

$$
\begin{aligned}
& X(m)=F(m, m-1) X(m-1)+W(m) \\
& Y(m)=H(m) X(m)+V(m)
\end{aligned}
$$

Among them: $\mathrm{F}(\mathrm{m}, \mathrm{m}-1)$ is the state transition matrix, $\mathrm{W}(\mathrm{m})$ is the state of zero mean Gaussian noise, $H(m)$ is the measurement matrix, $V(m)$ is a zero mean Gaussian noise measurement, and $\mathrm{W}(\mathrm{m})$ and $\mathrm{V}(\mathrm{m})$ statistically independent to each other.

\section{The test results and analysis}

Using the method of ray-tracing method, and the original scheme mean-shift to feature as the background of the aircraft tracking target image sequence test. Figure 1 as the target over the obstructions in the process of tracking test result, the white rectangle for the "target model" area of ray-tracing method, rectangular box center for tracking point location; And the white cross intersection of the original scheme mean-shift method trace point location, its size and white rectangle area the size of the region. Figure1(a) for target tracking area not obscured image; Figure1(b) is the target part into the woods, tracking area not obscured image tracking, kalman filter and Mean-shift jointly determine the trace "Target model" kernel function histogram is updated; Figure1(c) is the target completely into the woods, and is completely blocked, at this time "target model" nuclear function histogram is retained, completed by kalman filter target tracking; Figure1(d) is the target to fly out of the mask is still stable during the tracking. By shade trees in the aircraft in the process, the original scheme-shift method "target model" a serious interference, clutter appear frequently updated even excessively, makes it can't reflect the characteristics of aircraft target, lead to white cross fork that target tracking points stay on the branches, there was an error tracking. Thus, although in image sequences, the change of the target and background is bigger, but combined with kalman filter the grayscale average deviation of target tracking method is maintained in a particular area of the target, achieve a stable target tracking, target tracking under complex background is effective.
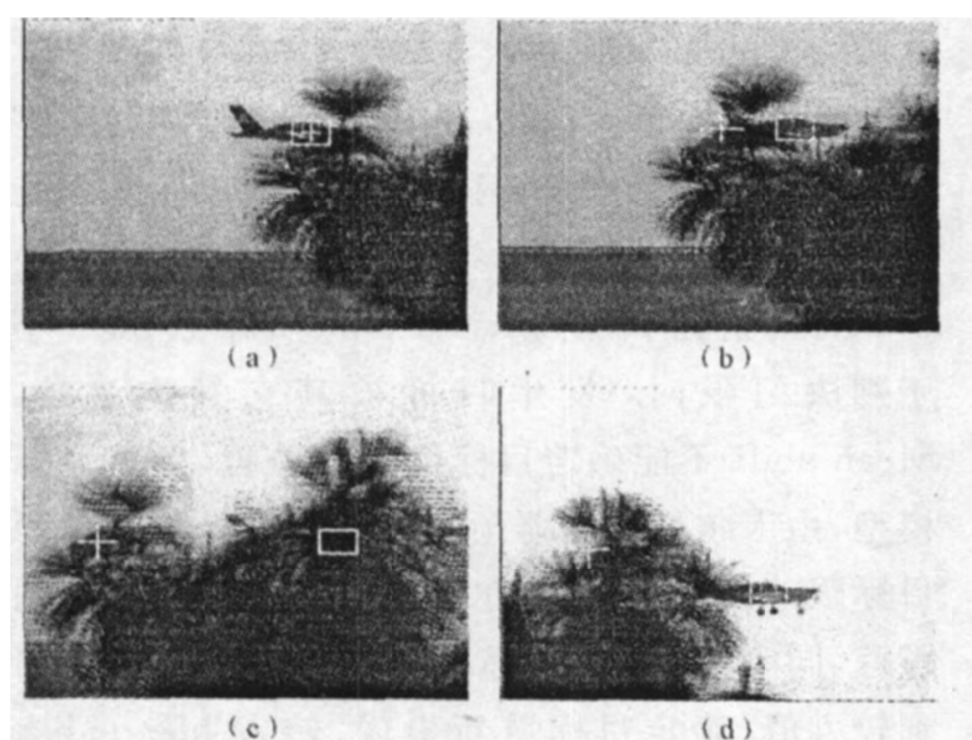

Figure1. Under the condition of target tracking results 


\section{Conclusion}

Analysis of the Mean-shift cannot very good tracking moving targets under complex background: the main reasons for Mean-shift algorithm is required to search the starting center should be close to the target location, otherwise it will lead to target tracking points stay on local extreme of similarity coefficient, cause target error tracking; Goals often be other objects in the scene, target model is background interference, leading to excessive update target model. According to the above problem, combine Mean-shift and kalman filter target tracking method using kalman filter to predict the target in the current moment of starting position, while Mean-shift looking for targets in the location of the neighborhood. At the same time, use Bhattacharyya coefficient measurement "target model" and "the candidate model" similarity, determine whether" candidate model "can be replaced as" target model ", to avoid the target model updating. Test show that the method in the target attitude and background change is bigger, even be scenery can still maintain the stability of the target tracking.

\section{Reference}

[1] Bovik, 1983, Generalization of median filtering using linear combinations of order statistics [J]. IEEE Trans. on Acoustics, Speech, 31(6): 1342-1350.

[2] Donoho DL, 1994, Ideal spatial adaptation via wavelet shrink age [J]. Biometrical, 81: 425-455.

[3] Geman D, 1992, Constrained restoration and there cover of discontinuities [J].IEEE. Trans. Pattern Analysis and Machine Intelligence, 14(3): 367-383.

[4] Liu Bin, 1999, Spectro gram enhancement algorithm: A soft thresholding based approach [J]. Ultrasound in Medical and Biology, 25(5): 839-846.

[5] Lin J H, 1989, Optimal stack filtering and the estimation and structural approaches to image processing [J]. IEEE Trans. on Acoustics, Speech, and Signal Processing, 37(12): 2037-2066.

[6] You Yuli, 2000, Fourth-order partial differential equations for noise removal [J]. IEEE Trans. Image Processing, 9(10): 1723-1730. 